STRUCTURAL BIOLOGY COMMUNICATIONS

ISSN 2053-230X

\section{Crystal structure of a family 6 cellobiohydrolase from the basidiomycete Phanerochaete chrysosporium}

\author{
Mikako Tachioka, ${ }^{a}$ Akihiko Nakamura, ${ }^{a} \ddagger$ Takuya Ishida, ${ }^{a} \S$ Kiyohiko Igarashi ${ }^{a, b}$ and \\ Masahiro Samejima ${ }^{a *}$
}

Received 31 March 2017

Accepted 31 May 2017

Edited by A. Nakagawa, Osaka University, Japan

₹ Current address: Institute for Molecular Science, National Institute of Natural Science, 5-1 Higashiyama, Myodaiji, Okazaki, Aichi 444-8787, Japan.

$\S$ Current address: EM Utilization Center, Human Spaceflight Technology Directorate, Japan Aerospace Exploration Agency (JAXA), 2-1-1 Sengen, Tsukuba-shi, Ibaraki-ken, 3058505 Japan,

Keywords: cellulases; Phanerochaete chrysosporium; cellobiohydrolase; biomass utilization; carbohydrate-active enzymes.

PDB references: PCCel6A, 5xcy; complex with cellobiose, $5 \times c z$

Supporting information: this article has supporting information at journals.iucr.org/f

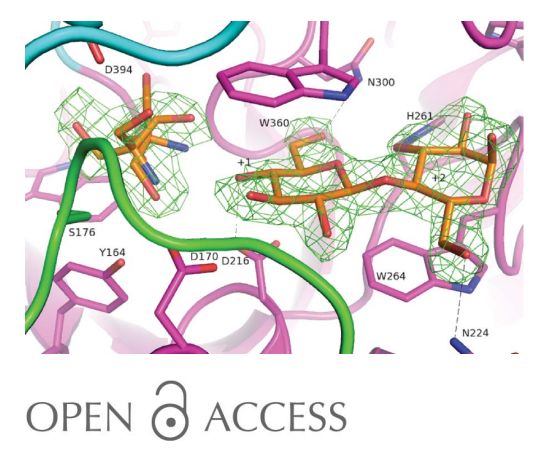

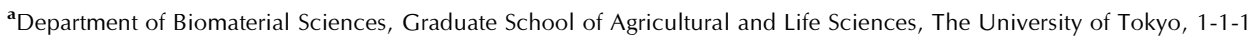
Yayoi, Bunkyo-ku, Tokyo 113-8657, Japan, and ${ }^{\mathbf{b}}$ VTT Technical Research Centre of Finland, PO Box 1000, Tietotie 2, Espoo FI-02044 VTT, Finland. *Correspondence e-mail: amsam@mail.ecc.u-tokyo.ac.jp

Cellobiohydrolases belonging to glycoside hydrolase family 6 (CBH II, Cel6A) play key roles in the hydrolysis of crystalline cellulose. CBH II from the whiterot fungus Phanerochaete chrysosporium (PcCel6A) consists of a catalytic domain (CD) and a carbohydrate-binding module connected by a linker peptide, like other known fungal cellobiohydrolases. In the present study, the $\mathrm{CD}$ of PcCel6A was crystallized without ligands, and $p$-nitrophenyl $\beta$-D-cellotrioside ( $p$ NPG3) was soaked into the crystals. The determined structures of the ligand-free and $p$ NPG3-soaked crystals revealed that binding of cellobiose at substrate subsites +1 and +2 induces a conformational change of the $\mathrm{N}$-terminal and $\mathrm{C}$-terminal loops, switching the tunnel-shaped active site from the open to the closed form.

\section{Introduction}

Cellulose is the most abundant biopolymer on Earth, and is a potential alternative resource to fossil-based fuels and chemicals. It consists of $\beta$-1,4-linked D-glucose units, and is mainly found in plant cell walls in highly crystalline forms, which provide mechanical strength and resistance to microbial and chemical breakdown. Cellulose-degrading microorganisms have evolved to produce an array of extracellular enzymes that convert cellulose into soluble oligosaccharides. Cellobiohydrolases (EC 3.2.1.91), which hydrolyze cellulose to cellobiose (a $\beta$-1,4-linked $\mathrm{D}$-glucose dimer), are key members of their enzyme cocktails, playing a major role in the hydrolysis of crystalline cellulose.

Glycoside hydrolase (GH) family 6, a class of enzymes defined by amino-acid sequence similarities in the CAZy includes cellobiohydrolases and endoglucanases (EC 3.2.1.4). Structural studies have revealed that cellobiohydrolases of this family have tunnel-like catalytic sites covered by flexible loops, which are not necessarily conserved in endoglucanases (Rouvinen et al., 1990; Spezio et al., 1993). This family is also known for its unusual 'Grotthus-type' inverting mechanism: the enzymes appear to lack a general base residue that would activate a catalytic water molecule for direct nucleophilic attack; instead, observations of ordered water molecules near the active site led to the proposal that an additional water molecule is involved in hydrolysis, serving to bridge between the catalytic water molecule and the proton-accepting residue (Koivula et al., 2002).

The GH family 6 cellobiohydrolase from the basidiomycete P. chrysosporium (PcCel6A) consists of a catalytic domain database (Carbohydrate-Active enZymes; http://www.cazy.org), 
(CD) and an N-terminal carbohydrate-binding module (CBM) connected by a linker region (Tempelaars et al., 1994); this modular structure is typical of fungal GH family 6 cellobiohydrolases (Mertz et al., 2005). In a previous study, we cloned and recombinantly expressed $P c$ Cel6A and found that the hydrolysis rate of $P c$ Cel6A was greatly accelerated by a polymorphic change of crystalline cellulose (Igarashi et al., 2012). PcCel6A has been the target of protein engineering aimed at improving its thermostability (Heinzelman et al., 2009; Ito et al., 2013), and we have also recently applied random mutagenesis to this enzyme (Tachioka et al., 2016). Here, we report the structure of the $\mathrm{CD}$ of $P c \mathrm{Cel} 6 \mathrm{~A}$ in its apo form and in complex with the ligand cellobiose.

\section{Materials and methods}

\subsection{Expression and purification}

Cloning of the cel6A gene from $P$. chrysosporium and the construction of the pPICZ $\alpha / c e l 6 A$ vector have been described in a previous report (Igarashi et al., 2012). Deletion of the $\mathrm{N}$-terminal CBM-linker region (residues 1-81) was performed by a combination of inverse PCR and DpnI treatment. The recombinant protein was produced in $P$. pastoris using a 51 jar fermenter, as reported previously (Igarashi et al., 2012), and was then ultrafiltered and concentrated using a Kvick Lab Cassette $100 \mathrm{kDa}$ and $5 \mathrm{kDa}$ (GE Healthcare, USA). The protein solution in $20 \mathrm{mM}$ sodium acetate buffer $\mathrm{pH} 5.0$ containing $1 \mathrm{M}$ ammonium sulfate was applied onto a Phenyl Toyopearl 650S column (Tosoh Corporation, Japan) equilibrated with the same buffer. The protein was eluted with a reverse gradient to $20 \mathrm{~m} M$ sodium acetate buffer $\mathrm{pH} 5.0$ and was then analysed by SDS-PAGE. Doublet bands were seen at approximately $37 \mathrm{kDa}$, which might reflect a difference in glycosylation. Therefore, the fractions with lower molecular weight were collected. These fractions were equilibrated against $20 \mathrm{mM}$ Tris- $\mathrm{HCl}$ buffer $\mathrm{pH} 8.0$ and applied onto a DEAE Toyopearl 650S column (Tosoh Corporation) equilibrated with the same buffer. The protein was eluted from the column with a linear gradient from 0 to $20 \mathrm{mM} \mathrm{NaCl}$. The purified protein was dialyzed into $5 \mathrm{mM}$ Tris- $\mathrm{HCl}$ buffer $\mathrm{pH}$ 7.5 containing $100 \mathrm{~m} M \mathrm{NaCl}$.

\subsection{Crystallization, data collection and structure solution}

Crystallization was performed by the sitting-drop vapourdiffusion method. The drops were formed by mixing $1 \mu \mathrm{l}$ $20 \mathrm{mg} \mathrm{ml}^{-1}$ protein solution with the same volume of a reservoir solution composed of $20 \%(w / v)$ polyethylene glycol $3350,200 \mathrm{~m} M$ calcium acetate, $50 \mathrm{~m} M$ acetate buffer $\mathrm{pH} 5.0$, $10 \%(w / v)$ 2-methyl-2,4-pentanediol. To introduce the ligand, $p$-nitrophenyl $\beta$-D-cellotrioside ( $p$ NPG3) powder was dissolved in mother liquor and a crystal was incubated in this solution for $10 \mathrm{~h}$ prior to data collection. X-ray diffraction data sets were collected using synchrotron radiation on beamlines BL5A and BL17A of the Photon Factory, High Energy Accelerator Research Organization (KEK), Tsukuba, Japan. The data sets were processed and scaled using the
Table 1

$\mathrm{X}$-ray data-collection and refinement statistics.

Values in parentheses are for the highest resolution shell.

\begin{tabular}{|c|c|c|}
\hline & $P c$ Cel6A & $P c$ Cel6A-cellobiose \\
\hline \multicolumn{3}{|l|}{ Data collection } \\
\hline Beamline & BL5A & BL17A \\
\hline Wavelength $(\AA)$ & 1.00000 & 0.98000 \\
\hline Space group & $P 2_{1} 2_{1} 2_{1}$ & $P 2_{1} 2_{1} 2_{1}$ \\
\hline \multicolumn{3}{|l|}{ Unit-cell parameters } \\
\hline$a(\AA)$ & 54.7 & 54.5 \\
\hline$b(\AA)$ & 67.2 & 67.0 \\
\hline$c(\AA)$ & 89.1 & 85.1 \\
\hline Resolution $(\AA)$ & $50.00-1.20(1.22-1.20)$ & $50.00-2.10(2.14-2.10)$ \\
\hline Total reflections & 1014578 & 129843 \\
\hline Unique reflections & 103410 & 19086 \\
\hline Completeness (\%) & $99.9(99.2)$ & $99.5(99.0)$ \\
\hline Multiplicity (\%) & $3.1(2.8)$ & $6.8(6.8)$ \\
\hline Average $I / \sigma(I)$ & $43.7(5.9)$ & $15.5(3.1)$ \\
\hline$R_{\text {merge }}(\%)$ & $5.5(28.8)$ & $7.2(48.4)$ \\
\hline Mosaicity range $\left({ }^{\circ}\right)$ & $0.26-0.32$ & $0.79-1.27$ \\
\hline \multicolumn{3}{|l|}{ Refinement } \\
\hline Resolution $(\AA)$ & $28.6-1.20(1.24-1.20)$ & $45.9-2.1(2.18-2.10)$ \\
\hline$R_{\text {work }}(\%)$ & $13.8(14.9)$ & $17.7(21.9)$ \\
\hline$R_{\text {free }}(\%)$ & 15.9 (19.6) & $23.5(27.6)$ \\
\hline No. of reflections & $103210(10094)$ & $18676(1806)$ \\
\hline No. of atoms & 3515 & 2950 \\
\hline \multicolumn{3}{|l|}{ R.m.s.d. from ideal values } \\
\hline Bond lengths $(\AA ̊)$ & 0.005 & 0.007 \\
\hline Bond angles $\left({ }^{\circ}\right)$ & 0.83 & 0.85 \\
\hline \multicolumn{3}{|l|}{ Ramachandran plot } \\
\hline Favoured regions (\%) & 96.9 & 97.0 \\
\hline Additionally allowed (\%) & 3.1 & 3.0 \\
\hline Outliers (\%) & 0 & 0 \\
\hline
\end{tabular}

HKL-2000 suite (Otwinowski \& Minor, 1997). The sequence of $P c$ Cel6A was submitted to the Phyre 2 web server (Kelley \& Sternberg, 2009; http://www.sbg.bio.ic.ac.uk/phyre2) to obtain a suitable search model for molecular replacement with MOLREP, an auto-MR function in the CCP4 suite (Winn et al., 2011). Manual model rebuilding and refinement were performed using Coot (Emsley et al., 2010) and PHENIX (Adams et al., 2010). Data-collection and refinement statistics are shown in Table 1. Most of the molecular-graphics images were prepared using PyMOL (v.1.7; Schrödinger). The relative $B$ factor was calculated by dividing the average $B$ factor of each residue by that of the whole protein: $11.6 \AA^{2}$ for the apo structure and $20.0 \AA^{2}$ for the PcCel6A-cellobiose structure. The apo and cellobiose-bound structures were superposed using the phenix.superpose_maps tool, and the r.m.s.d. values between all $\mathrm{C}^{\alpha}$-atom pairs and the relative $B$-factor values were visualized using UCSF Chimera (http://www.cgl.ucsf.edu/ chimera; Pettersen et al., 2004). Hydrophobicity was visualized using the color_h script in PyMOL.

\section{Results and discussion}

3.1. Overall structure of the PcCel6A catalytic domain

$\mathrm{X}$-ray data sets were collected from a $P c$ Cel6A crystal and a $p$ NPG3-soaked crystal to 1.2 and $2.1 \AA$ resolution, respectively. Both crystals belonged to space group $P 2_{1} 2_{1} 2_{1}$ with one molecule in the asymmetric unit, and the structures were 
refined to $R_{\text {work }}$ and $R_{\text {free }}$ values of 13.8 and $15.9 \%$ and of 17.7 and $23.5 \%$, respectively.

The CD of $P c$ Cel6A consists of a distorted seven-stranded $\beta / \alpha_{8}$-barrel, like the CDs of other fungal GH family 6 cellobiohydrolases (Fig. 1). The final structures included residues $82-439$. The catalytic site is located in a tunnel enclosed by a pair of loops (residues 174-178 and 390-425), designated here as the $\mathrm{N}$-terminal and $\mathrm{C}$-terminal loops (shown in green and blue, respectively, in Fig. 1). A structure-similarity search of the Protein Data Bank (PDB) using the DALI server (Holm \& Rosenström, 2010) revealed highly homologous structures among GH family 6 enzymes. The overall structure and ligandbound structure of $P c$ Cel6A showed the greatest similarity to Cel6A from Coprinopsis cinerea (CcCel6A; Tamura et al., 2012; PDB entry $3 \mathrm{vog}$ ), with an r.m.s.d. of $0.7 \AA$ for $357 \mathrm{C}^{\alpha}$ atoms and a $Z$-score of 60.8 , and to $C c$ Cel6A- $p$ NPG3 (PDB entry $3 \mathrm{voi}$ ), with an r.m.s.d. of $0.9 \AA$ for $357 \mathrm{C}^{\alpha}$ atoms and a $Z$-score of 61.3 , respectively. The sequence similarity between $P c$ Cel6A and $C c$ Cel6A was $65 \%$. The catalytically important residues and two conserved disulfide bridges (Cys171-Cys230 and Cys361-Cys408) are shown in Supplementary Fig. S1, together with sequence alignments of $P c$ Cel6A, CcCel6A,
Cel6A from Trichoderma reesei ( $\operatorname{TrCel6A)}$ and Cel6A from Humicola insolens (HiCel6A). The $\mathrm{CD}$ of PcCel6A has one potential $N$-glycosylation site at Asn398 according to the NetNglyc 1.0 Server (http://www.cbs.dtu.dk/services/NetNGlyc/), but no electron density owing to sugars was visible in the structures, indicating that the proteins used to prepare the crystals were nonglycosylated.

\subsection{Ligand binding at the $+1 /+2$ sites and conformational change of loops}

The ligand-bound structure obtained from the $p$ NPG3soaked crystal contained one $\alpha$-cellobiose molecule, which occupies subsites +1 and +2 in the substrate-binding cleft (Fig. 2). The observed $\alpha$-cellobiose is considered to be a product of the hydrolysis of $p$ NPG3 under the crystallization conditions at $\mathrm{pH} 5$. This is plausible because the GH family 6 enzymes perform hydrolysis of $\beta$-1,4-glycosidic bonds with inversion of anomeric configuration, and are known to cleave chromophoric cello-oligosaccharides to produce cellobiose units (Claeyssens et al., 1989). The cleavage pattern of $p$ NPG3 in this structure is different from the $C c$ Cel6A structure, in

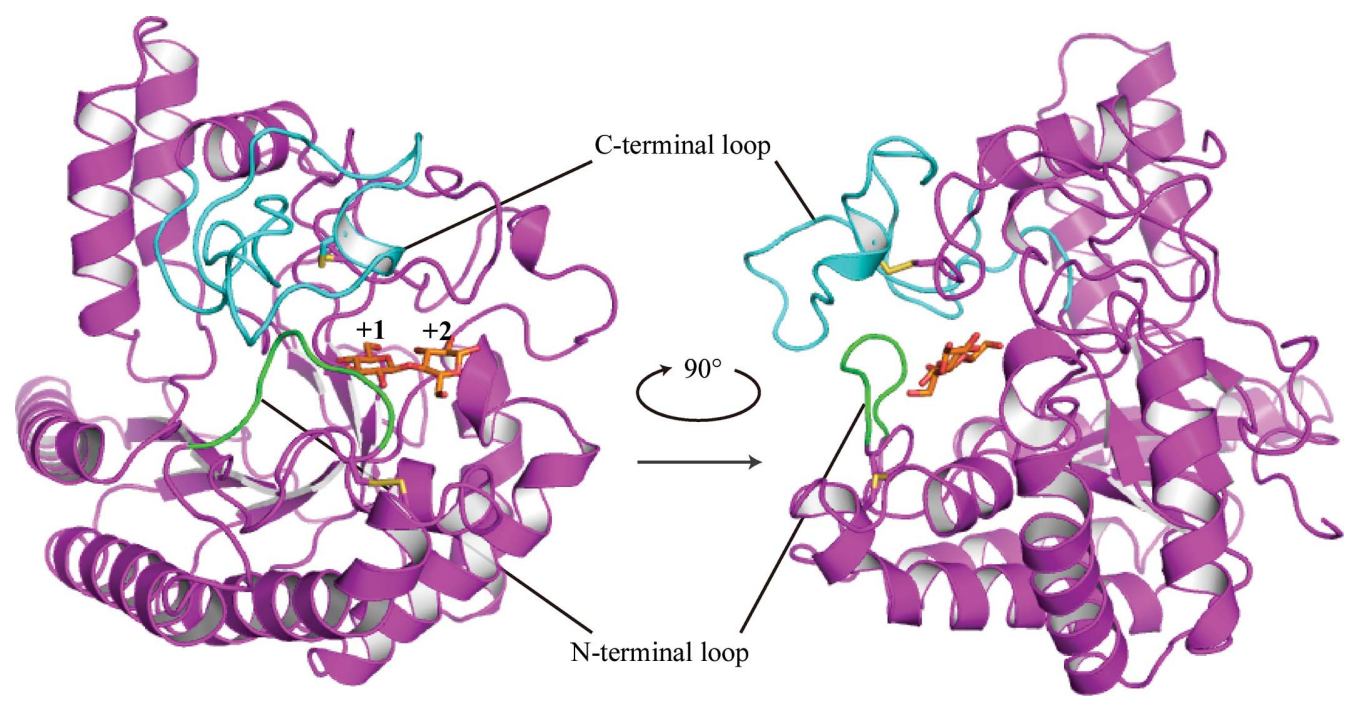

Figure 1

Overall structure of $P c$ Cel6A with cellobiose bound at subsites +1 and +2 . The N-terminal and C-terminal loops covering the catalytic centre are coloured green and cyan, respectively.

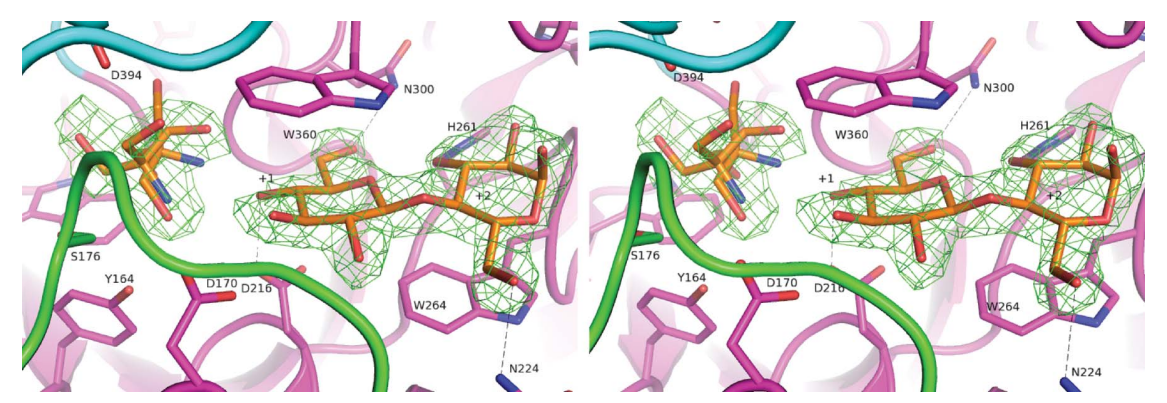

Figure 2

Close-up view of subsites +1 and +2 of $P c$ Cel6A with cellobiose as a ligand. The $\left|F_{\mathrm{o}}\right|-\left|F_{\mathrm{c}}\right|$ map was calculated without ligand atoms and contoured at the $3 \sigma$ level. The Tris molecule at subsite -1 was modelled in a double conformation. 
which nonhydrolyzed $p$ NPG 3 bound at subsites +1 to +4 (PDB entry 3voi; Tamura et al., 2012), and from the $C c$ Cel6C structure, in which two $p$ NPG2 molecules bind at subsites -3 to -1 and +1 to +3 (PDB entry 3abx; Liu et al., 2010).

As in other GH family 6 enzymes, the -1 subsite is often occupied by molecules other than waters and sugars, such as cations and low-molecular-weight compounds (Supplementary Table S1), and an unmodelled electron-density blob was similarly observed at the -1 site in the present liganded structure. An octahedrally coordinated $\mathrm{Mg}^{2+}$ ion found at subsite -1 of the $C c$ Cel6C- $p$ NPG3 structure (PDB entry $3 \mathrm{voi}$ ), where the ligands only occupied plus-numbered subsites as in this $P c$ Cel6A-cellobiose complex, led us to initially place a hydrated $\mathrm{Ca}^{2+}$ ion into the density blob. However, the $B$ factor of the hydrated $\mathrm{Ca}^{2+}$ ion $\left(48.8 \AA^{2}\right)$ was quite high compared with those of cellobiose $\left(18.5-26.8 \AA^{2}\right)$ and the water molecules around the subsite (approximately 15-30 $\AA^{2}$ ). The automated ligand-identification tool in PHENIX (Terwilliger et al., 2006) predicted Tris as the most favourable of the molecules in the crystallization condition, and the $B$ factors for a single Tris molecule were in a reasonable range (29.1-33.4 $\AA^{2}$ ). The second conformation of the Tris molecule was placed manually into the residual electron density of this model, and a molecule with multiple conformations appears to be the most probable explanation, with $B$ factors of 18.3$24.8 \AA^{2}$. The Tris molecule made hydrogen bonds to O4 of glucose at subsite +1 , the OD1 atom of Tyr164, the NZ atom of Lys388, the carbonyl O atom and the OD1 atom of Asp394 in addition to several water molecules. No water molecule corresponding to the catalytic water was found in the structure because of the binding of the putative Tris molecule.

The tunnel-enclosing N- and C-terminal loops of GH family 6 enzymes have been observed in several conformations in previous crystallographic studies (reviewed in Payne et al., 2015). Two major conformations of the loops are known, an 'open' conformation that makes the subsites more accessible and a 'closed' conformation for arrangement of the catalytic site residues into the catalytically competent configuration. In the cellobiose-bound structure, the loops adopted the closed conformation, in contrast to the open conformation observed in the apo structure. As summarized in Supplementary Table S1, the loops may adopt either conformation in enzymes for which the structures are known. The occupation of +1 and +2 sites by sugar moieties results in the closure of the loops, with the exception that proton-accepting residues are 'off' conformation and interact with serines on the loop, as in $H i$ Cel6A structures where sugars bind in upside-down configurations (PDB entries 1oc5, 1 oc7 and 1ocj; Varrot et al., 2003).

\subsection{Noncatalytic but notable residues of PcCel6A}

Mutagenesis work performed on $P c$ Cel6A revealed several properties of noncatalytic residues of this enzyme (see Heinzelman et al., 2009; Ito et al., 2013; Tachioka et al., 2016). In our recent work, the substitution mutation $\mathrm{W} 267 \mathrm{C}$ was found to be a critical mutation for the degradation of crystalline cellulose

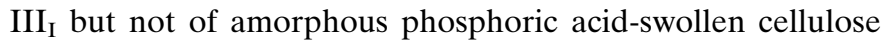
(Tachioka et al., 2016), which was a similar finding to the work on $\operatorname{TrCel6A}$ (Koivula et al., 1998). Trp267 is located at the entrance to the catalytic site at subsite +4 and probably plays a specialized role in the recognition and recruitment of a single cellulose chain from the crystalline surface (Fig. 3a). Heinzelman and coworkers successfully engineered $P c$ Cel6A to improve its thermostability by a remarkable $10^{\circ} \mathrm{C}$ by a single mutation of Cys393 to serine (Heinzelman et al., 2009). This free cysteine makes a weaker hydrogen bond to the carbonyl of Pro418, with a distance of about $3.3 \AA$ (Fig. $3 b$ ), than the serine residues that are found in other homologous fungal structures which originally have a serine instead of cysteine (2.6-2.7 $\AA$ in Chaetomium thermophilum Cel6A and ${ }_{c c}$ Cel6C), which is consistent with their discussion. Ito and coworkers also improved the thermostability of $P c$ Cel6A by 16 cumulative mutations (Ito et al., 2013). The largest stabilization of $1.2^{\circ} \mathrm{C}$ was achieved by a mutation of Met257 to isoleucine, and the authors deduced from modelling PcCel6A that an exchange to more hydrophobic residues stabilizes the

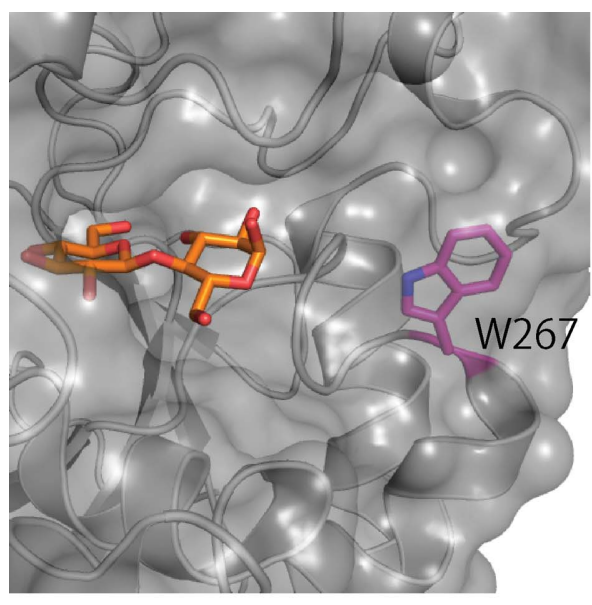

(a)

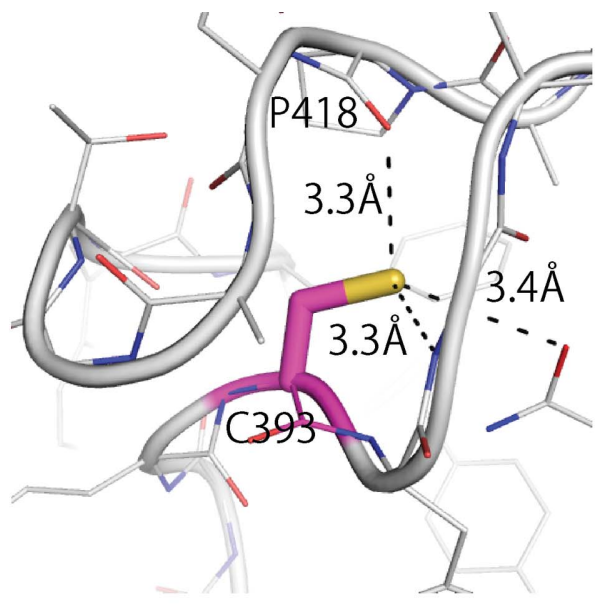

(b)

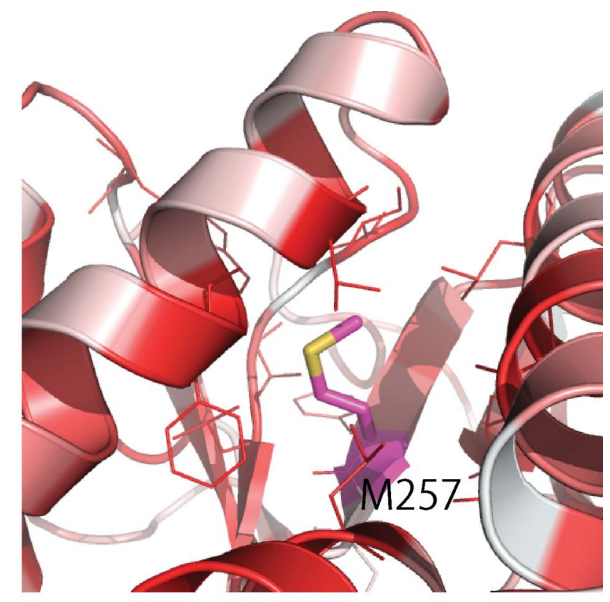

(c)

Figure 3

Enlarged views of Trp267 (a), Cys393 (b) and Met257 (c). Each residue is coloured magenta. The red-white colour in $(c)$ represents the hydrophobicity of the residues. 


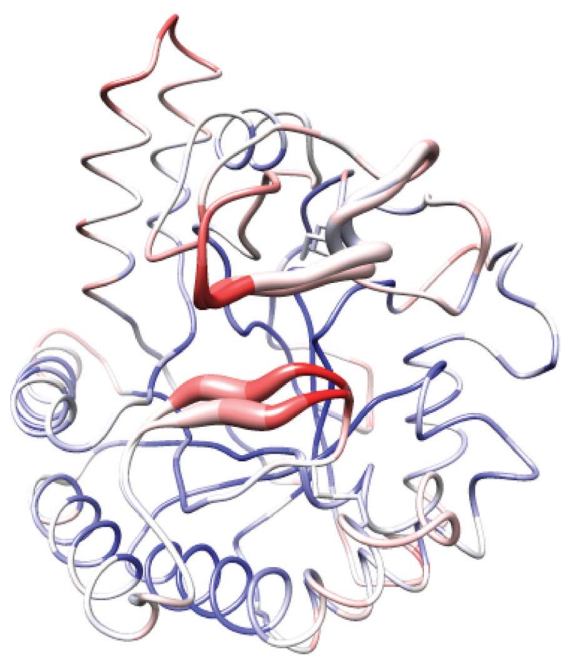

(a)

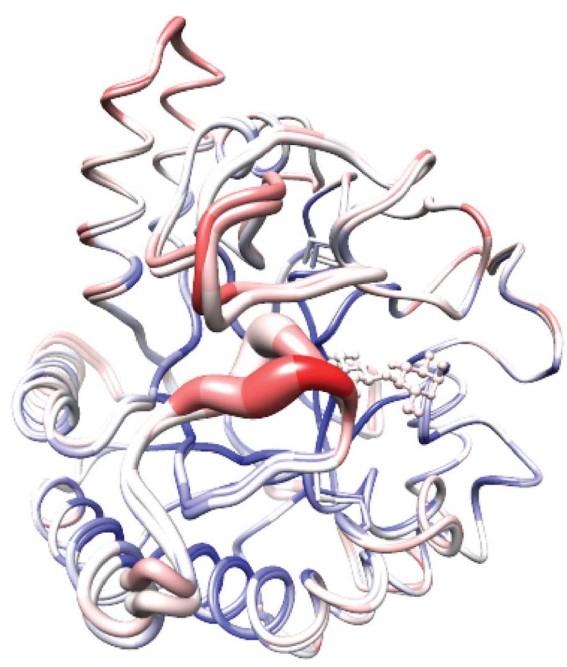

(b)

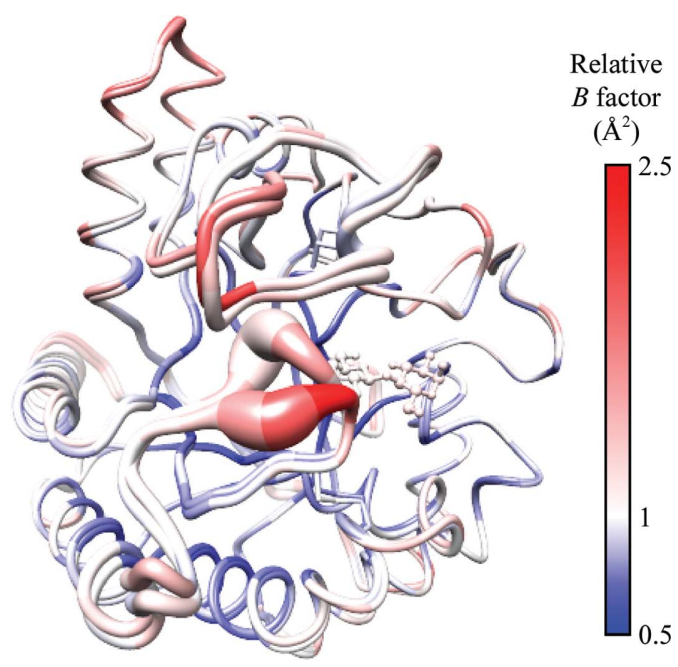

(c)

Figure 4

Superposition of the apo and cellobiose-bound structures of PcCel6A. (a) The apo structure was observed in multiple conformations: the 'relatively open' and 'most open' conformations are shown. (b) Superposition of the relatively open apo structure and the cellobiose-bound structure. (c) Superposition of the most open apo structure and the cellobiose-bound structure. The width of the ribbon in each figure represents the r.m.s.d. value between the superposed conformations. The relative $B$ factors were calculated for each apo and cellobiose-bound structure.

structure in its buried protein environment. Our results reveal that Met257 is surrounded by hydrophobic side chains of $\alpha$-helix and supports their findings (Fig. 3c).

\subsection{Loop flexibility}

The flexibility and mobility of the loops are important for the catalytic activity. As noted above in $\$ 3.2$, the loops of $P c$ Cel6A remained open in the absence of bound ligand, but occupation of the substrate-binding sites induced conformational change of the loops, resulting in a narrowing of the active-site cleft. To further examine the situation, the relative $B$ factors of residues and r.m.s.d. values among the observed conformations of PcCel6A were visualized by means of a blue-red colour scale and worm representations, respectively (Fig. 4). The residues in the loops have consistently high $B$ factors compared with other regions of the protein. As shown in Fig. 4(a), the $\mathrm{N}$ - and C-terminal loops of the PcCel6A apo structure were both modelled as double conformations with similar occupancies ranging from 0.46 to 0.54 . In Figs. $4(b)$ and $4(c)$, the relatively open and most open apo structures are compared with the cellobiose-bound structure, respectively. The most dynamic conformational change occurred in the $\mathrm{N}$-terminal loop concomitantly with ligand binding, as shown by a ribbon representation, the thickness of which is in proportion to the r.m.s.d values between two conformations. In the C-terminal loop region, on the other hand, the r.m.s.d. values between the apo and cellobiose-bound structures were similar to that between the two conformations of the apo structure. Therefore, both loops showed clear flexibility, but their mobility and response to ligand binding were different.

Although the role of each loop in GH family 6 cellobiohydrolases is not fully understood, it has been suggested that a conformational change of the loops would occur during the catalytic cycle (see Payne et al., 2015). In this study, we determined both the open and closed structures of the CD of $P c$ Cel6A. Careful analysis of the flexibility and mobility of the $\mathrm{N}$-terminal and C-terminal tunnel-enclosing loops revealed differences in their characteristics, and we speculate that the loops are involved in both the chemical hydrolysis reaction of a single polysaccharide chain and the physical interaction with the crystalline surface of cellulose. The present structural information should afford insight into the structure-function relationship and provide a basis for more detailed mechanistic studies of this industrially and scientifically important enzyme.

\section{Acknowledgements}

We thank Dr Shinya Fushinobu for the provision of beam time and all beamline staff members at the Photon Factory for their kind help with data collection. We are also grateful to Dr Jerry Ståhlberg at the Swedish University of Agricultural Sciences for valuable discussions and suggestions. This work was performed under the approval of the Photon Factory Program Advisory Committee (Proposal Nos. 2013G191 and 2013G194).

\section{Funding information}

This research was supported by a Grant-in-Aid for Innovative Areas from the Japanese Ministry of Education, Culture, Sports and Technology (MEXT) to KI (Nos. 24114001 and 24114008), a Grant-in-Aid for Scientific Research from the Japan Society for the Promotion of Science (JSPS) to KI (B, No. 24380089), an Impulsing Paradigm Change through Disruptive Technologies (ImPACT) from the Japan Science and Technology Agency (JST) to KI and a Grant-in-Aid for JSPS Fellows from JSPS to MT (15J10657).

\section{References}

Adams, P. D. et al. (2010). Acta Cryst. D66, 213-221. 
Claeyssens, M., Van Tilbeurgh, H., Tomme, P., Wood, T. M. \& McRae, S. I. (1989). Biochem. J. 261, 819-825.

Emsley, P., Lohkamp, B., Scott, W. G. \& Cowtan, K. (2010). Acta Cryst. D66, 486-501.

Heinzelman, P., Snow, C. D., Smith, M. A., Yu, X., Kannan, A., Boulware, K., Villalobos, A., Govindarajan, S., Minshull, J. \& Arnold, F. H. (2009). J. Biol. Chem. 284, 26229-26233.

Holm, L. \& Rosenström, P. (2010). Nucleic Acids Res. 38, W545W549.

Igarashi, K., Maruyama, M., Nakamura, A., Ishida, T., Wada, M. \& Samejima, M. (2012). J. Appl. Glycosci. 59, 105-110.

Ito, Y., Ikeuchi, A. \& Imamura, C. (2013). Protein Eng. Des. Sel. 26, 73-79.

Kelley, L. A. \& Sternberg, M. J. E. (2009). Nature Protoc. 4, $363-$ 371.

Koivula, A., Kinnari, T., Harjunpää, V., Ruohonen, L., Teleman, A., Drakenberg, T., Rouvinen, J., Jones, T. A. \& Teeri, T. T. (1998). FEBS Lett. 429, 341-346.

Koivula, A. et al. (2002). J. Am. Chem. Soc. 124, 10015-10024.

Liu, Y., Yoshida, M., Kurakata, Y., Miyazaki, T., Igarashi, K., Samejima, M., Fukuda, K., Nishikawa, A. \& Tonozuka, T. (2010). FEBS J. 277, 1532-1542.

Mertz, B., Kuczenski, R. S., Larsen, R. T., Hill, A. D. \& Reilly, P. J. (2005). Biopolymers, 79, 197-206.
Otwinowski, Z. \& Minor, W. (1997). Methods Enzymol. 276, 307-326. Payne, C. M., Knott, B. C., Mayes, H. B., Hansson, H., Himmel, M. E., Sandgren, M., Ståhlberg, J. \& Beckham, G. T. (2015). Chem. Rev. 115, 1308-1448.

Pettersen, E. F., Goddard, T. D., Huang, C. C., Couch, G. S., Greenblatt, D. M., Meng, E. C. \& Ferrin, T. E. (2004). J. Comput. Chem. 25, 1605-1612.

Rouvinen, J., Bergfors, T., Teeri, T., Knowles, J. K. C. \& Jones, T. A. (1990). Science, 249, 380-386.

Spezio, M., Wilson, D. B. \& Karplus, P. A. (1993). Biochemistry, 32, 9906-9916.

Tachioka, M., Sugimoto, N., Nakamura, A., Sunagawa, N., Ishida, T., Uchiyama, T., Igarashi, K. \& Samejima, M. (2016). Biotechnol. Biofuels, 9, 1-10.

Tamura, M., Miyazaki, T., Tanaka, Y., Yoshida, M., Nishikawa, A. \& Tonozuka, T. (2012). FEBS J. 279, 1871-1882.

Tempelaars, C. A. M., Birch, P. R. J., Sims, P. F. G. \& Broda, P. (1994). Appl. Environ. Microbiol. 60, 4387-4393.

Terwilliger, T. C., Klei, H., Adams, P. D., Moriarty, N. W. \& Cohn, J. D. (2006). Acta Cryst. D62, 915-922.

Varrot, A., Frandsen, T. P., von Ossowski, I., Boyer, V., Cottaz, S., Driguez, H., Schülein, M. \& Davies, G. J. (2003). Structure, 11, 855-864.

Winn, M. D. et al. (2011). Acta Cryst. D67, 235-242. 